\title{
Study of Downlink Radio Resource Allocation Scheme with Interference Coordination in LTE - A Network
}

\author{
Yen-Wen Chen and Chen-Ju Chen
}

\begin{abstract}
Carrier Aggregation (CA) is one of the most important technologies in the LTE-Advanced (LTE-A) system for better transmission efficiency. The basic idea of $\mathrm{CA}$ is to adopt more than one component carrier (CC) for user equipment (UE) to increase its bandwidth. However, when UE adopts component carriers from other adjacent base stations, it will cause the interference and may decrease transmission efficiency especially for the cell edge UE. Therefore, solving interference with carrier aggregation is a critical issue toward the effective improvement of system throughput. In this paper, we propose a novel method to deal with not only the interference between pico station and macro base station, but also the interference between base stations. In the proposed scheme, the radio interference, channel condition of $\mathrm{UE}$, and load balance are taken into consideration for proper CC selection so as to improve the cell-edge UE performance. The proposed scheme considers the interaction and correlation between Inter-cell Interference Coordination (ICIC) and enhanced Inter-cell Interference Coordination (eICIC) to arrange the secondary CC for LTE-A UE. The simulation results show that the proposed scheme can increase transmission efficiency especially for the edge $U E$.
\end{abstract}

Index Terms-Carrier aggregation, component carrier, inter-cell interference coordination, long term evolution-advanced.

\section{INTRODUCTION}

Recently, the fast deployment of mobile multimedia services pushes the high demand of bandwidth in wireless networks. The mobile device can access the radio channel through either the unlicensed band, e.g. WiFi, or licensed band, such as long term evolution (LTE), for internet connections. Generally, the unlicensed band provides easy and unrestricted for users access, however, it is hard to maintain the efficient spectrum utilization due to the unmanageable accesses from users. On the contrarily, the licensed band provides the well-defined control protocol for the control node, e.g. the eNodeB in LTE network, to manage the radio resource. However, the radio interference always happens unpredictably and it may decrease the spectrum efficiency not only in the unlicensed band networks but also the licensed band networks. In order to minimize the radio interference, especially for the manageable licensed band, the

Manuscript received February 5, 2017; revised April 4, 2017. This work was supported in part by the Ministry of Science and Technology (MOST) (grant numbers: 105-2221-E-008-031-MY2, and 105-2221-E-008-030), Taiwan.

Yen-Wen Chen is with the Department of Communication Engineering, National Central University, Taiwan (e-mail: ywchen@ce.ncu.edu.tw).

Chen-Ju Chen is with the Department of Communication Engineering, National Central University, Taiwan. She is also with MediaTek Inc., Taiwan (e-mail: julie081217@gmail.com). negotiation mechanism is required among interference devices. In LTE, the inter cell interference (ICI) is one of the most severe factors that downgrade the transmission quality and system throughput. To get over the ICI problem has become one of the most important issues toward effective transmission in cellular networks. Recently, several studies were proposed for resource allocation with carrier aggregation in LTE [1]-[3]. However, most of them only considered the interference between the pluralities of pico stations and the associated macro base station. The interference among macro base stations was not well discussed. Generally, the pico stations are deployed over the cell edge area for the compensation of poor radio condition of the associated macro base station, however, the neighbor macro base stations may introduce radio interference to this area. Thus it may not always a proper decision to choose the radio channel of this small cell without considering the interference from the other stations.

In order to provide bandwidth for users, 3GPP organization evolves the utilization of radio resource from time division duplex (TDD) and frequency division duplex (FDD) to carrier aggregation (CA) since 3 GPP release 10 as shown in Fig. 1 [4]. Comparing to the technologies of LTE and LTE-A, LTE-A provides UE the capability of aggregating more than component carrier for higher throughput. And, therefore, the interference coordination issue becomes more critical than that in LTE environment. Because UE needs to select the $\mathrm{CC}$, which has better channel condition to it, however, it may interfere with the channel chosen by other UE. In this paper, we study and propose the method to deal with the interference between base stations about the CC selection by taking the load of $\mathrm{CC}$ and the channel condition of the UE received in different $\mathrm{CC}$ s into consideration so as to improve the cell-edge UE performance.

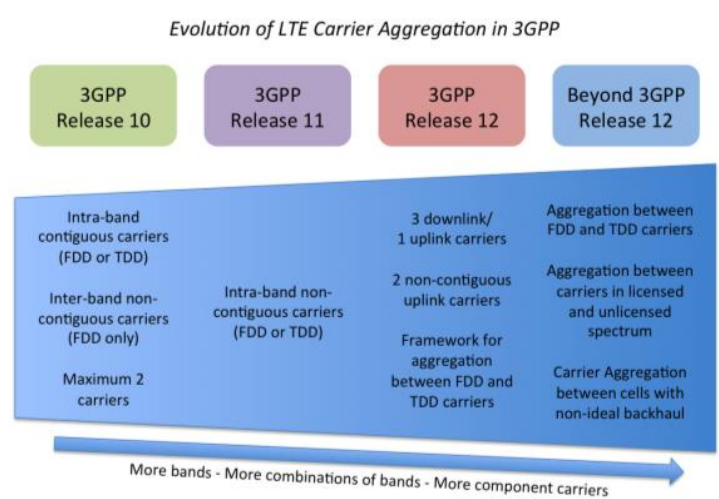

Fig. 1. The evolution of 3GPP [4]

The rest of this paper is organized as follows. The following section provides the related works of our study 
issue. The integrated scheme for Inter-cell Interference Coordination (ICIC) and enhanced Inter-cell Interference Coordination (eICIC) are proposed in section III. The simulation results are illustrated with discussion in section IV. And, the final section concludes our works.

\section{RELATED WORKS}

The ICIC and eICIC mechanisms were provided to reduce the interferences between macro base stations and between macro base station and pico station, respectively. In LTE, eNBs exchange information to each other through the X2 interface for management purpose. Thus eNB can negotiate with other eNBs for radio resource utilization according to the received Relative Narrowband Tx Power (RNTP), and send the High-Interference Indicator (HII), and Overload Indicator (OI) to/from its neighbor eNBs as reference when performing resource allocation as well as handover [5].

For the ICIC schemes, the proposed scheme in [6] deals with the ICIC issue based on Harmony Search (HS). According to their procedure, eNBs perform decentralized arbitration to mute $\mathrm{RBs}$ by referring to the average interference weight calculated by each eNB. In [7], the author suggested to assign conflicted RBs by comparing the traffic load of two adjacent macro base stations. Its concept is to let eNB, which has heavier traffic load, have higher probability to obtain the conflicted RB. However, the impact of channel condition was not well considered.

In order to increase overall throughput, the reuse of valuable spectrum is one of the convincible solutions towards this objective. Thus pico stations or remote radio head (RRH) units can be deployed over the cell edge of the macro station. Due to the low transmission power of the pico station, there is no interference among pico stations and the spectrum can then be reused. However, the interference issue between the macro base station and its associated small stations exists. And the framework of eICIC was proposed in 3GPP release 10. The basic concept of eICIC is that eNB adopts the almost blank sub-frames (ABS) so that pico stations can utilize those sub-frames without interference from the base station. Although macro base station will decrease its throughput, several pico stations can reuse these sub-frames and, therefore, the overall throughput increases. As shown in Fig. 2 , the macro base station mutes the sub-frames 2 and $n-2$ so that pico base stations 1 and 2 can utilizes these two sub-frames without interference from macro base station. .

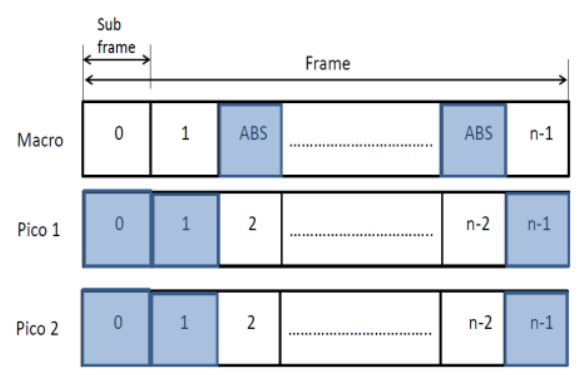

Fig. 2. The ABS of macro base station for eICIC.

The CA is applied to increase the bandwidth in LTE-A system. Several researches discussed the allocation and scheduling of CC to maximize system throughput [8], [9]. In addition to the LTE-sim simulator, three scheduling algorithms, including general multi-band scheduling, basic multi-band scheduling, and enhanced multi-band scheduling, were proposed to arrange the resource by using weight factor [8]. In [9], the logarithmic utility function and the sigmoidal-like utility function were designed to allocate the resource block $(\mathrm{RB})$ of $\mathrm{CC}$ in a proportional fairness manner. Additionally, the improvement of the throughput for cell edge users was discussed in [10]-[12]. In [10], the authors proposed the user association algorithm to decide whether UE is in the cell edge or not. Then the greedy binary carrier selection algorithm was applied for CC selection. In [11], the interferences of control channel and data channel were analyzed separately, and the price based algorithm was designed to determine the transmission power so that the interference can be minimized. In addition to interference aware scheduler, the requirements of quality of services (QoS) for different traffic types, e.g. data and video, were taken into consideration in [12]. The interference can be subdivided into frequency domain and time domain. In [13], the dynamic Q-learning and satisfaction based learning approaches were proposed for the decisions of bias, transmission power, and primary CC.

The above proposed schemes focus on the negotiation between the macro base station and the pico base stations, the interference from other neighbor macro base stations is not well discussed. Therefore, it is the main research objective of this paper.

\section{THE PROPOSED INTEGRATED ICIC AND EICIC SCHEME}

The LTE-A UE shall have a fixed primary CC (PCC) for the connection management and control signaling and may choose other secondary CC (SCC) to increase bandwidth. As each UE may have different channel condition in different $\mathrm{CC}$ and the bandwidth of each CC is limited, therefore, the selection of SCC is complex and critical for spectrum efficiency and system throughput. In addition to the channel condition, the interference shall also be properly considered so that the spectrum can be effectively utilized. Basically, ICIC and eICIC negotiate the spectrum in the frequency domain among macro base stations and in the time domain between macro base station and pico base station, respectively, as shown in the following Fig. 3.

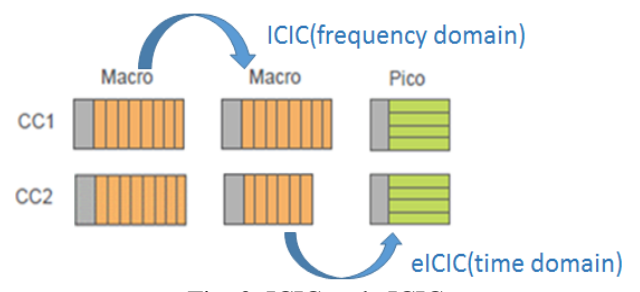

Fig. 3. ICIC and eICIC.

Basically, UE shall select PCC at the connection establish stage and PCC is fixed during the connection duration and most control signals between UE and eNB go through PCC. UE shall report its channel conditions, such as channel quality indicator (CQI) and RNTP, of the feasible eNBs to its management eNB through PCC. The SCC can be flexibly adjusted according to the received channel conditions. The 
management eNB shall decide the handover, modulation and coding scheme (MCS), the selection of SCC, etc., through PCC. The proposed scheme is subdivided into the SCC selection phase and the resource allocation phase as described in the following.

The selection of PCC for each LTE-A UE is basically according to the signal to noise ratio, however, the interference from other $\mathrm{CC}$ is considered as shown in equation (1). $\gamma_{u, c, k}$ in equation (1) denotes the received SINR value of the $u$-th UE from the CC $k$ of eNB $c$. The higher value of $\gamma_{u, c, k}$ means the more suitable for the $u$-th $\mathrm{UE}$ selects the CC $k$ of eNB $c$ as its CC.

$$
\gamma_{u, c, k}=\frac{P_{u, c, k}}{\sum_{C_{I} \neq c} P_{u, C_{I}, k}+N_{0}}
$$

where $P_{u, c, k}$ means the received power of the $u$-th UE from the $\mathrm{CC} k$ of eNB $c$. The proposed scheme will always assign the $\mathrm{CC} k$ of eNB $c$ to the $u$-th UE as its PCC for the highest value $\gamma_{u, c, k}$ of each UE if the bandwidth of $\mathrm{CC} k$ is affordable. It is noted that, at this stage, eNB only allocates one RB of the PCC for each UE. If the required bandwidth of $\mathrm{UE}$ is not satisfied, the proposed scheme will choose the suitable CC, which may be its PCC or the other CC, for further bandwidth allocation. The procedure keeps allocation until either the bandwidth is satisfied or there is no bandwidth left in the CC. It is noted that the number of CC of UE is limited and this is the constraint during the bandwidth allocation. For example, if each UE can accommodate two CCs, then one CC shall be PCC and it can only have one SCC. Once its SCC is decided, it can only be allocated with bandwidth from these two $\mathrm{CC}$. The basic flow of the proposed scheme is provided in the following Fig. 4.

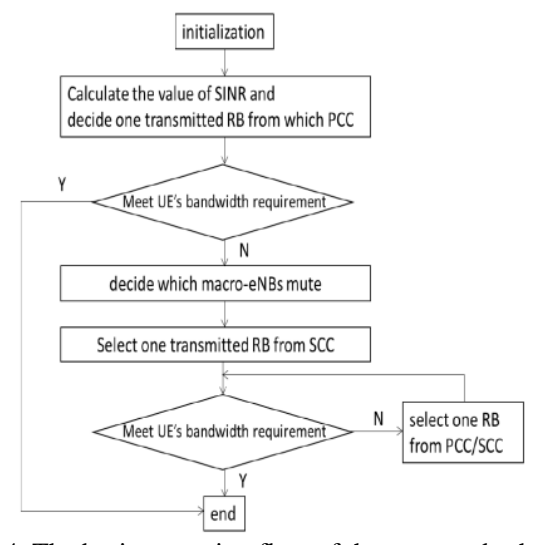

Fig. 4. The basic operation flow of the proposed scheme.

In Fig. 4, the selection of one RB from either PCC or SCC also refers to the $\gamma_{u, c, k}$ value calculated in equation (1). Thus, if the original assigned CC of the PCC has the highest value, the allocated RB will come from the original PCC if it still has RB left, otherwise the other $\mathrm{CC}$ will be selected as the SCC of this UE. If the selected CC comes from pico cell, then the proposed scheme will perform eICIC at time domain. In the proposed scheme, as the pico eNB is always deployed over the cell edge of the macro cell and it may be interfered by more than one macro cell, then we need to decide which macro eNB shall mute some sub-frames so that the pico cell can have better transmission performance. In the proposed scheme, we apply the weighting function to decide which macro cell shall mute. The basic concept of the weighting function $W_{i}$ of macro cell $i$ refers the numbers of UEs that can be served by one macro cell, two macro cells, or pico cell to maximize the system utilization as shown in equation (2).

$$
W_{i}=\frac{N_{1,0}^{i}}{N_{2,1}^{i}} \times \frac{1}{\sum_{k=0}^{x-1} R_{k}^{i}}
$$

where $N_{m, n}^{i}$ means the number of UE, which under macro cell $i$, that can be covered by $m$ macro cells and $n$ pico cells, and $R_{k}^{i}$ denotes the number of residual RBs of the $k$-th CC of the macro cell $i$. In the proposed scheme, UE can be located at the location, where is covered by either one, two, or three macro base stations. And the proposed scheme assumes that the pico cell is deployed over the cell edge, however, except the area covered by three macro base stations as shown in Fig. 5. As the weighting function is designed to determine which macro base station shall mute for eICIC, therefore, we only compare the numbers of UEs that are only served by one macro base station and are located at the coverage of two macro base stations and the pico base station in equation (2). Thus $N_{1,0}^{i}$ and $N_{2,1}^{i}$ of equation (2) denote the numbers of UEs for the above deployment assumption.

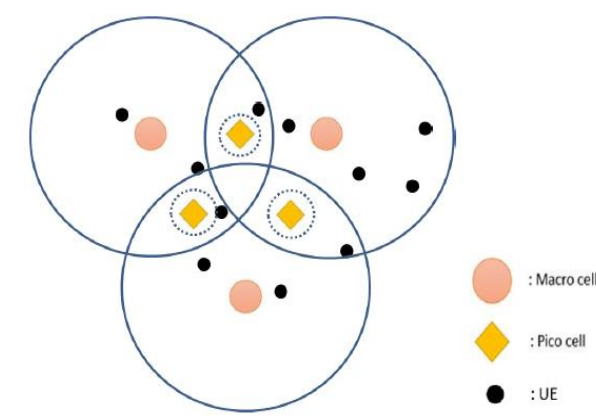

Fig. 5. Deployment of macro base cells and pico cells.

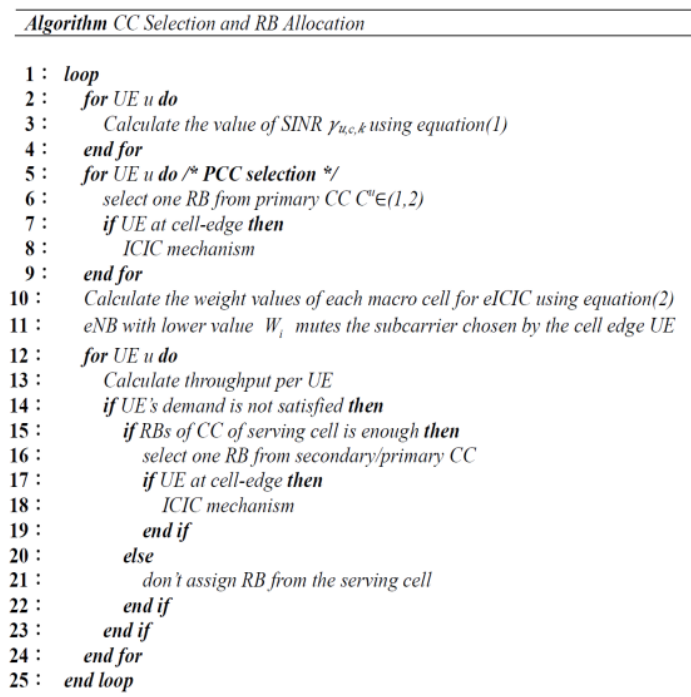


The higher value of $W_{i}$ means that the macro base station $i$ need keep more RBs to be allocated for the UE which can only be served by it because of the smaller values of $N_{2,1}^{i}$ and $R_{k}^{i}$. The proposed CC selection and resource allocation algorithm is illustrated as follows.

\section{EXPERIMENTAL SimULATIONS}

In order to investigate the performance of the proposed scheme, exhaustive simulations were performed. The architecture that consists of three macro base stations and three pico base stations is applied during the simulations. Each base station was assumed to have two $\mathrm{CC}$ and the maximum number of $\mathrm{CC}$ can be aggregated by each UE was also assumed to be 2. The cell edge area means the intersection between macro cells. And the widest distance of the cell edge is $200 \mathrm{~m}$. The TDD with configuration 5 configuration was adopted and $8 \mathrm{~ms}$ of each frame was reserved for downlink transmission. Therefore, there are 800 RBs (or 272 RBGs) to be utilized. The other simulation parameters are given in the following Table I.

TABLE I: SIMULATION PARAMETERS

\begin{tabular}{|c|c|}
\hline Carrier bandwidth & $10 \mathrm{MHz}$ \\
\hline Number of cell & $\begin{array}{c}\text { Macro cell:3 } \\
\text { Pico cell:3 }\end{array}$ \\
\hline ISD/cell radius & Macro cell:500m ; Pico cell:40m \\
\hline Carrier frequency & $10 \mathrm{MHz} @ 1.8 \mathrm{GHz}$ \\
\hline Number of RB & 50 \\
\hline Size of RBG & 3 \\
\hline Number of RBG & 17 \\
\hline BS transmit power & $\begin{array}{c}\text { Macro cell:46 dBm } \\
\text { Pico cell:30 dBm }\end{array}$ \\
\hline Antenna height & $15 \mathrm{~m}$ \\
\hline & $\begin{array}{c}\text { Macro cell: } \\
\text { Path loss model }[14]\end{array}$ \\
& $\begin{array}{c}\mathrm{dB}(R[\mathrm{~km}]) \\
\text { Pico cell: }\end{array}$ \\
& $140.7+21 \log (\mathrm{F} / 2000)+36.7 \log (\mathrm{R}) \mathrm{dB}(R[\mathrm{~km}])$ \\
\hline $\begin{array}{c}\text { UE bandwidth } \\
\text { requirements }\end{array}$ & $3 \mathrm{Mbps}$ \\
\hline ABS ratio & $0 / 8,1 / 8,3 / 8,5 / 8$ \\
\hline
\end{tabular}

This paper focuses on the suitable CC selection and the harmonic coordination of ICIC and eICIC, therefore, we compare the results of 6 simulation scenarios as follows:

- Scenario 1 (proposed): ICIC and eICIC

- Scenario 2 (w/o ICIC): neither ICIC nor eICIC

- Scenario 3 (w/o eICIC): only ICIC

- Scenario 4 (fix muting 2,3): ICIC and eICIC, fix muting by eNB 2 and eNB 3 (i.e. $W_{i}$ is not applied)

- Scenario 5 (fix muting 1,3): ICIC and eICIC, fix muting by eNB 1 and eNB 3

- Scenario 6 (fix muting 1,2): ICIC and eICIC, fix muting by eNB 1 and eNB 2

The simulation results of the average throughputs of all UEs and the cell edge UEs are shown in Fig. 6 and 7, respectively. It shows that the results of scenario 2 (w/o ICIC) is the worst. Thus the average throughput mainly depends on whether ICIC is applied or not.

The minimum bandwidth requirement of each UE is assumed to be $3 \mathrm{Mbps}$, we compare the dissatification ratios, i.e. the ratio of $\mathrm{UE}$ that can not receive $3 \mathrm{Mbps}$, of the six scenarios in Fig. 8. It indicates that the scenario 2 has the lower dissatisfaction ratio when the number of UEs exceeds 80. The main reason is that the coverage of the overlaping area between macro base stations and the pico cell is relatively much smaller than that of the macro cell (40m v.s. $500 \mathrm{~m}$ in radius) and the UEs are uniformally distributed over the area. The ICIC mechanism is utilized to tradeoff the utilization of $\mathrm{RB}$ at the cell edge. Although the scenario 2 does not take care of the UE in the cell edge, it can satisfy most UEs.

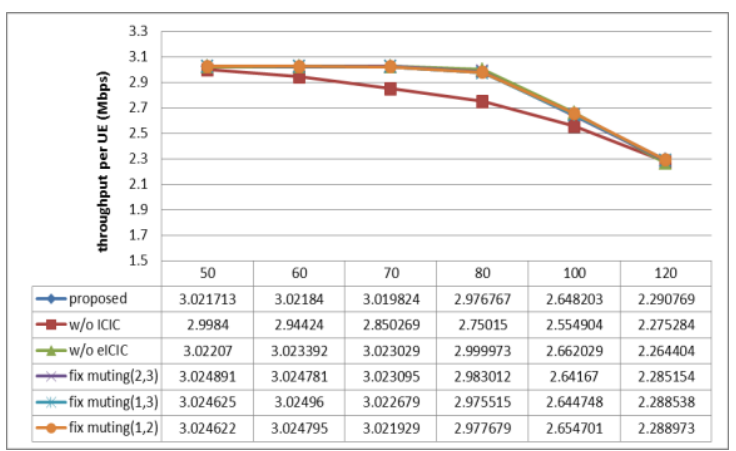

Fig. 6. The average overall throughput

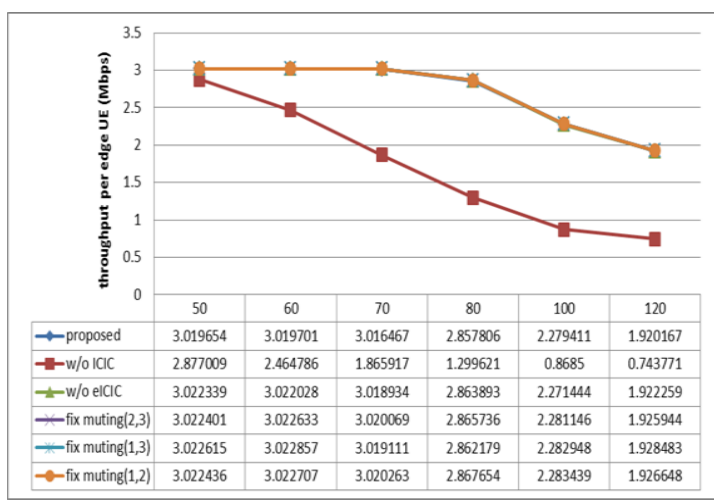

Fig. 7. The average cell edge throughput.

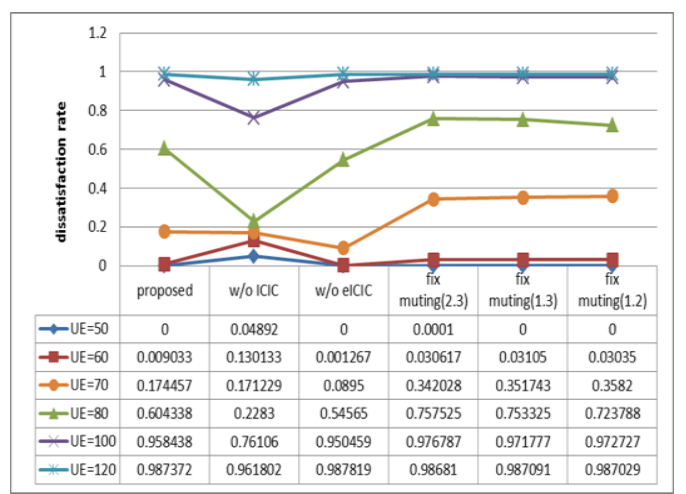

Fig. 8. Comparison of dissatification ratios.

The above simulations assume that the UE is uniformly distributed over the simulation area. In order to examine the performance when the distribution of UE is nonuniform, we put $20 \%, 50 \%$, and $80 \%$ of UEs to be located in the cell edge to see their performance of throughput. We compare the simulation results of the scenario 1 and scenario 3 (i.e. ICIC only) to examine the effectiveness of eICIC. Fig. 9 presents the average throughputs of cell edge UEs, respectively. It clearly illustrate that the proposed scheme (scenario 1) achieves higher throughput for cell edge UEs in the unbalance UE distribution condition especially when the 
number of UEs is getting more.

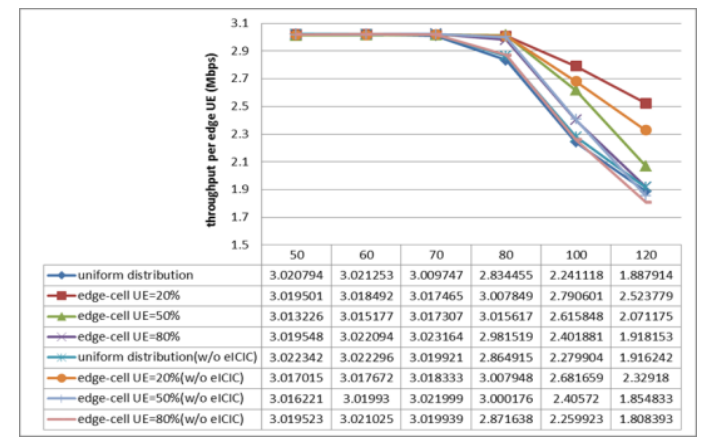

Fig. 9. The average cell edge throughput for unbalance number of UE.

The simulation results of 100 UEs by applying different ABS ratio are given in Fig. 10. The results clearly indicate that the cell edge throughput can be significantly improved if cell edge UE is dense and the higher ABS ratio is applied.

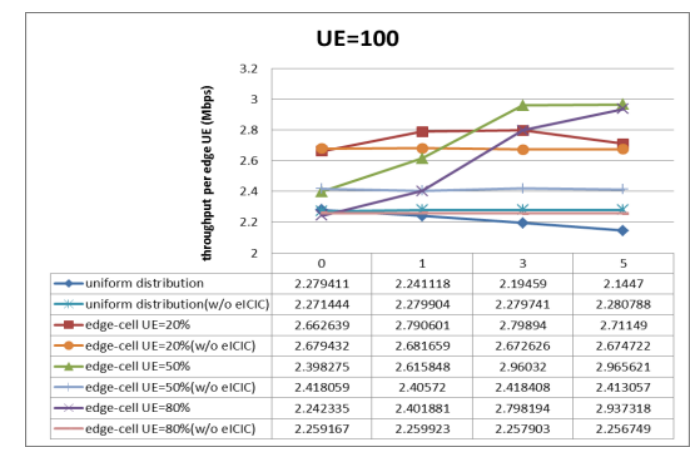

Fig. 10. The average cell edge throughput of different ABS ratio.

According to the above simulation results, the performance is affected by interference coordination mechanism and UE distribution. We summarize the pros and cons of the policities discussed in the paper in the following the following Table II.

TABLE II: PROS AND CONS OF DIFFERENT INTERFERENCE POLICIES

\begin{tabular}{|c|c|}
\hline ICIC+eICIC & $\begin{array}{c}\text { Pros: significant improvement on edge UE throughput and } \\
\text { the other UEs can still maintain in acceptable level } \\
\text { Cons: helpless when most UEs are located in macro cell }\end{array}$ \\
\hline w/o ICIC & $\begin{array}{c}\text { Pros: benefit to overall throughput for banance and } \\
\text { undense UE distribution } \\
\text { Cons: unfair and lower throughput when load is heavier }\end{array}$ \\
\hline w/o eICIC & $\begin{array}{c}\text { Pros: better performance when less UE in pico cell } \\
\text { Cons: unable to mitigate the load of macro-eNB }\end{array}$ \\
\hline $\begin{array}{c}\text { Fixed } \\
\text { muting }\end{array}$ & $\begin{array}{c}\text { Pros: benefit to cell edge UE } \\
\text { Cons: poor utilization of RB in macro cells }\end{array}$ \\
\hline
\end{tabular}

\section{CONClusions}

In this paper, we propose the integrated interference coordination scheme for proper radio resource allocation in LTE-A network. The performance of the proposed scheme is evaluated through exhaustive simulations. In addition to the simulation results, we analyze the effectiveness of the coordination policies, which include ICIC, eICIC, and ABS ratio. The results illustrate that the proposed scheme can effectively utilize radio resource so as to improve the throughput of cell edge UE. However, the ABS ratio shall be carefully adjusted according to the number and distribution of UE. And the dynamic ABS adjustment scheme will be one of our future research directions.

\section{REFERENCES}

[1] 3GPP TS 36.101 (v12.5.0 Release12), "Evolved universal terrestrial radio access (E-UTRA); User equipment (UE) radio transmission and reception," November 2014.

[2] 3gpp. [Online]. Available: (June 2013). http://www.3gpp.org/technologies/keywords-acronyms/101-carrier-ag gregation-explained

[3] G.-X. Yuan et al., "Carrier aggregation for LTE-advanced mobile communication systems," IEEE Communication Magazine, vol. 48, no. 2, pp. 88-93, February 2010.

[4] A. Brydon. (March 2014). Evolution of LTE-advanced carrier aggregation. [Online]. Available: http://www.unwiredinsight.com/2014/lte-carrier-aggregation-evolutio $\mathrm{n}$

[5] 3GPP TS 36.213 (v12.5.0 Release 12), "Evolved universal terrestrial radio access (E-UTRA); physical layer procedures," March 2015.

[6] S. S. Khalifa et al., "Inter-cell interference coordination for highly mobile users in lte-advanced systems," Vehicular Technology Conference (VTC Spring), pp. 1-5, June 2013.

[7] S.-J. Wang et al., "A decentralized downlink dynamic ICIC method for multi-cell OFDMA system," in Proc. International Conference on Wireless Communications and Signal Processing (WCSP), pp. 1-5, November 2011.

[8] D. Robalo et al., "Extending the LTE-sim simulator with multi-band scheduling algorithms for carrier aggregation in LTE-advanced scenarios," in Proc. IEEE 81st Vehicular Technology Conference (VTC Spring), pp.1-6, May 2015.

[9] H. Shajaiah et al., "Utility proportional fairness resource allocation with carrier aggregation in 4G-LTE," in Proc. IEEE Military Communication Conference, pp. 412-417, November 2013.

[10] C.-Y. Sun et al., "Component carrier selection and interference coordination for carrier aggregation system in heterogeneous networks," in Proc. IEEE 14th International Conference on Communication Technology (ICCT), pp. 402-407, November 2012.

[11] H.-L. Jiang et al., "Carrier aggregation based interference coordination for lte-a macro-pico HetNet," in Proc. IEEE 77th Vehicular Technology Conference (VTC Spring), pp. 1-6, June 2013.

[12] Z. Limani et al., "Interference-aware resource scheduling in lte hetnets with carrier aggregation support," in Proc. IEEE International Conference on Communication (ICC), pp. 3137-3142, June 2015.

[13] M. Simsek et al., "Learning based frequency- and time-domain inter-cell interference coordination in HetNets," in Proc. ieee transaction on vehicular technology, vol. 64, no. 10, pp. 4589-4602, October 2015.

[14] H. Wang et al., "Uplink inter-site carrier aggregation between macro and small cells in heterogeneous networks," in Proc. IEEE 80th Vehicular Technology Conference (VTC Fall), pp.1-5, September 2014.

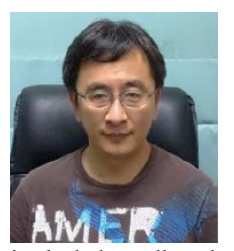

Yen-Wen Chen received the Ph.D. degree from the Electronic Engineering, National Taiwan University of Science and Technology (NTUST) in 1997. He worked at Chunghua Telecommunication Laboratories Taiwan during 1983 to 1998 . Currently, he is a professor of the department of Communication Engineering, National Central University, Taiwan. His research interests include broadband mobile networks, QoS management, network applications, cloud services, and software defined networking (SDN). Dr. Chen is a member of the IEEE communication society.

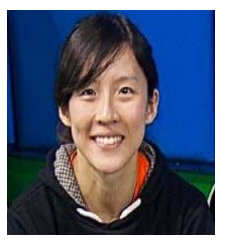

Chen-Ju Chen received the BS and MS degrees from the Department of Communication Engineering, National Central University, Taiwan. And, currently, she is the research engineer of MediaTek Inc., Taiwan. 